\title{
Retinopatia hipertensiva: Revisão
}

\author{
Hypertensiveretinopathy:Review
}

\author{
Aurélio Paulo Batista da Silva ${ }^{1}$ \\ Andréa Vasconcellos Batista da Silva ${ }^{2}$ \\ Fernando Luiz Herkenhof $f^{3}$
}

${ }^{1}$ Professor Assistente do Departamento de Morfologia da Universidade Federal do Espírito Santo. Pós-graduação nível mestrado do Programa de Pós-graduação em Ciências Fisiológicas, Universidade Federal do Espírito Santo, Centro Biomédico.

2 Professora de Anatomia da Faculdade Salesiana de Vitória.

${ }^{3}$ Professor Adjunto do Departamento de Fisiologia da Universidade Federal do Espírito Santo. Programa de Pós-Graduação em Ciências Fisiológicas, Universidade Federal do Espírito Santo, Centro Biomédico.

Endereço para correspondência: Rua Dr. Eurico de Aguiar, 415 - Apto. 606 - Vitória (ES) CEP 29055-280.

E-mail: apbatista@escelsa.com.br

Recebido para publicação em 03.12.2001

Aceito para publicação em 13.05.2002

\section{RESUMO}

O presente estudo faz uma revisão do tema retinopatia hipertensiva. Para tanto propôs-se uma breve revisão dos dados históricos da retinopatia hipertensiva. Este estudo relata as alterações clássicas da retinopatia hipertensiva e suas classificações, bem como os achados mais recentes associados à hipertensão arterial sistêmica, os prováveis mecanismos fisiopatológicos e as alterações histológicas associadas à retinopatia hipertensiva. Abordamos, ainda, os diversos métodos utilizados para a investigação, suas vantagens e desvantagens; uma visão crítica da interpretação dos sinais do envolvimento do bulbo ocular pela hipertensão arterial sistêmica; ainda, baseado na diversidade das metodologias de investigação da retinopatia, comenta-se a repercussão desta, na prevalência da retinopatia hipertensiva e suas implicações, como órgão-alvo da hipertensão arterial sistêmica, em um contexto atualizado da síndrome metabólica e de outros fatores associados à fisiopatologia da HAS, como a leptina e a endotelina.

Descritores: Hipertensão ocular/complicações; Doenças retinianas/etiologia; Pressão arterial; Oftalmoscopia/métodos

\section{INTRODUÇÃO}

O olho, formado por um conjunto de estruturas transparentes, (córnea, humor aquoso, lente e humor vítreo) é o único órgão do corpo humano ${ }^{(1)}$ que permite a observação direta da microvascularidade, uma “janela natural”"(2). O leito vascular, observado no fundo de olho, é constituído por vasos de pequeno calibre (arteríolas e vênulas) envolvidos no aumento da resistência vascular periférica, observada na hipertensão arterial sistêmica (HAS) ${ }^{(1)}$.

A literatura ${ }^{(3-6)}$ retrata as primeiras observações descritas do comprometimento ocular pela HAS: Richard Bright* , em 1836, observou o envolvimento ocular em pacientes com doença renal; mais tarde, por volta de 1850, com a descoberta do oftalmoscópio por Helmholtz ${ }^{* *}$, foi possível ratificar as observações feitas no passado e então, as alterações oftalmológicas, associadas a esta enfermidade, foram caracterizadas. Liebreich ${ }^{* * *}$, em 1859, descreveu as alterações do fundo de olho na HAS maligna (doença de Bright), designando-as de retinite albuminúrica. Nesta oportunidade foi observada a presença de hemorragia, exsudato e anormalidades arteriolares. Em 1855, von Graefe ${ }^{* * * *}$ descreveu o descolamento seroso da retina na toxemia

*Bright R. Cases and observations, illustrative of renal disease accompanied with the secretion of albuminous urine. Guys Hosp Rep 1836;1:338-400 apud ${ }^{(3)}$; **Helmholtz H. Beschreibung eines AugenSpiegels: zur Untersuchung der Netzhaut im lebenden Auge. Berlin:A. Förstner'sche, 1851apud ${ }^{(3)}$; ***Liebreich R. Ophthalmoskopischer Befund bei Morbus Brightii. Albrecht von Graefes Arch Ophthalmol 1859; 5:265-8 apud ${ }^{(4)} ;{ }^{* * * *}$ Von Graefe A. Mittheilungen vermischten inhalts. 1) Beobachtungen über Accommodation bei Linsendefekt, Muskelkrankheiten und Anomalieen der Iris. Albrecht von Graefe Arch Ophthalmol 1855; 2:187-94 apud $^{(3)}$ 
gravídica. Marcus Gunn, em $1898^{* * * * *}$, descreveu uma série de alterações retinianas (estreitamento e irregularidade das arteríolas retinianas) em pacientes com evidências de insuficiência vascular cerebral e/ou doença renal. Fishberg e Oppenheimer, em $1930^{* * * * * *}$, utilizaram o termo neuro-retinopatia hipertensiva para descrever as alterações de fundo de olho em pacientes com HAS e, posteriormente, consagrou-se o termo retinopatia hipertensiva (RH). Em 1904, Elschnig descreveu manchas no fundo de olho em pacientes com nefrite severa, que as denominou manchas de Elschnig. A primeira classificação da RH surgiu, em $1939^{(7)}$ com o trabalho de Keith, Wagener e Barker (KWB). Após esta, várias outras classificações foram propostas: com o componente hipertensivo e o componente arteriosclerótico foram avaliados separadamente ${ }^{(8)}$; às classificações de Waganer-Clay-Gipner, que se basearam, praticamente, no estreitamento arteriolar para classificar a RH; a de Scheie, também se baseou na diferenciação das alterações hipertensivas e arterioloescleróticas; e a de Leishman, que classificou a RH em sete grupos, levando em consideração, além das alterações hipertensivas e arterioloescleróticas, a idade do paciente ${ }^{(5)}$; foi ainda proposto $^{(9)}$ a união dos grupos 1 e 2 da classificação de KWB como uma nova classificação da RH. Isto, devido a uma difícil distinção entre estes dois grupos; outros pesquisadores ${ }^{(10)}$ utilizaram, na metodologia a classificação de Pyig Solanes, uma classificação também qualitativa; a classificação da Organização Mundial de Saúde foi utilizada ${ }^{(11)}$ para estudar a retinopatia hipertensiva em pacientes com nefropatia hipertensiva; a classificação de Hogan para a RH demonstra sua validade pela diferenciação dos sinais de esclerose vascular ocorrida com a idade, que não foi bem explorada na classificação de $\mathrm{KWB}^{(4)}$. Mais recentemente foi proposto classificar as alterações oftalmológicas da HAS maligna, de acordo com a ordem de aparecimento dos sinais, definindo três categorias: $\mathrm{RH}$, coroidopatia hipertensiva $(\mathrm{CH})$ e neuropatia óptica hipertensiva $(\mathrm{NOH})$, sendo que, a $\mathrm{CH}$ e a $\mathrm{NOH}$ não apresentaram diferença no momento do aparecimento das lesões as quais caracterizaram o seu envolvimento ${ }^{(12)}$.

\section{SINAIS DA RETINOPATIA HIPERTENSIVA}

A esclerose arteriolar, observada pelo aumento do reflexo arteriolar, pode adquirir o aspecto de fio de cobre e fio de prata; o cruzamento arteríolo-venular; o estreitamento do calibre arteriolar em grau variável, a hemorragia retiniana, o exsudato duro, as manchas algodonosas e o papiledema são os sinais clássicos de RH. Acrescenta-se a estes, a retificação e a tortuosidade arteriolar como sinais de $\mathrm{RH}^{(9)}$. $\mathrm{O}$ edema de retina, a obstrução arterial e venosa também foram citados como

\footnotetext{
${ }^{* * * * *}$ Gunn, M. On ophthalmoscopic evidence of general arterial disease. Trans Ophthalmol. Soc. U.K. 1898; 18:356-81 apud ${ }^{(5)}$; ${ }^{* * * * * *}$ Fishberg AM, Oppenheimer BS The differentiation and significance of certain ophthalmoscopic pictures in hypertensive disease. Arch Intern Med 1930;46:901-20 apud ${ }^{(6)}$
}

sinais de $\mathrm{RH}^{(4)}$. No passado, o comprometimento da coróide foi descrito como as estrias de Siegrist e as manchas de Elschnig. O comprometimento vascular da coróide pela HAS tem sido investigado mais recentemente por outros autores ${ }^{(3,13-14)}$. O reflexo luminoso dos vasos, mesmo tradicionalmente associado à arteriosclerose, é sensível às alterações da pressão arterial (PA) ${ }^{(15)}$, necessitando de uma reavaliação na sua interpretação e na sua utilidade para a classificação da RH. Por outro lado, foi reportado ${ }^{(16)}$ a importância de se concentrar nas irregularidades do calibre vascular e no cruzamento arteríolovenular, ignorando outros sinais associados à HAS. A neuropatia óptica isquêmica é considerada por alguns autores ${ }^{(2,17-18)}$ uma forma de apresentação da RH. Atenção especial deve ser dada para o cuidado com a redução da PA em pacientes com neuropatia óptica isquêmica, devido à possível instalação de uma cegueira permanente ${ }^{(17)}$. A transudação periarteriolar intra-retiniana focal (Focal intraretinal periarteriolar transudates-FIPTs) foi descrita mais recentemente ${ }^{(19)}$. Este achado nada mais é que o edema retiniano perivascular que poderia falsear um estreitamento arteriolar ${ }^{(20)}$. Alterações no leito vascular retiniano, por meio da análise do diâmetro vascular após a sua bifurcação, e das alterações do ângulo de bifurcação entre os vasos têm sido investigados ${ }^{(21)}$. Tais alterações foram associadas à idade do paciente e, possivelmente, à HAS. Estes autores questionaram, também, a relevância destes achados na patogênese de doenças vasculares e da HAS. A hemorragia e microaneurismas retinianos são lesões relativamente freqüentes em idosos e significativamente relacionados à presença e severidade da $\mathrm{HAS}^{(22)}$.

\section{CLASSIFICAÇÃO DA RH DE KEITH-W AGENER-BARKER}

Em 1939 foi apresentado um estudo ${ }^{(7)}$ comparando as alterações do leito vascular do fundo de olho de 219 indivíduos hipertensos, não tratados, submetidos à biópsia do músculo peitoral maior. Este músculo foi selecionado pelo fato de ser o tecido muscular de maior representação no corpo humano e também por ser de fácil acesso. Desta forma, foram observadas as características histológicas vasculares deste tecido, comparado-o às alterações observadas no fundo de olho, as quais foram classificadas em quatro grupos (Tabela 1). Do grupo I ao grupo IV, quando comparadas às alterações histológicas do músculo peitoral maior, foi observado um comprometimento vascular mais intenso, com progressiva redução da taxa lúmen/espessura da parede do vaso, e também a presença

\footnotetext{
Tabela 1. Classificação da retinopatia hipertensiva de KWB

Grupo I Estreitamento arteriolar e alteração do reflexo arteriolar leves

Grupo II Estreitamento arteriolar e alteração do reflexo arteriolar mais acentuado e cruzamento arteríolo-venular

Grupo III Alterações do Grupo II, hemorragia retiniana e exsudatos

Grupo IV Alterações do Grupo III e papiledema
} 
de outras alterações histológicas, como: o aumento do número e do tamanho do núcleo das células musculares da parede do vaso; aumento do número e do tamanho do núcleo na íntima das arteríolas; trombos, evidências de arterite e periarterite, indicado pela evidenciação de linfócitos e fibroblastos na camada média e adventícia da parede do vaso. Com esta classificação, foi possível observar a progressão da $\mathrm{RH}$, bem como traduzir a condição vascular de outros órgãos-alvo da HAS, e, ainda, associar aos grupos da classificação, à severidade da HAS e à sobrevida do paciente. Cabe ressaltar que, os próprios autores sugerem uma análise cautelosa dos achados das arteríolas musculares, uma vez que as alterações não são sempre consistentes e não há uma correlação definitiva, entre a divisão dos quatro grupos com os achados das arteríolas musculares, a severidade clínica da HAS, ao tempo de vida após a biópsia do músculo e as alterações arteriolares retinianas. Exemplifica esta condição relatada anteriormente, comentando que alguns pacientes do grupo IV da classificação de KWB, com alterações características arteriolares retinianas, apresentavam discretas alterações nas arteríolas musculares.

\section{FISIOPATOLOGIA E HISTOPATOLOGIA DA RETINOPATIA HIPERTENSIVA}

A RH foi descrita em fase vasoconstritora, exsudativa, esclerótica e nas complicações da fase esclerótica ${ }^{(23)}$. Na fase vasoconstritora, há resposta vascular ao aumento da pressão arterial que se faz pelos mecanismos miogênicos e metabólicos. Isto porque, aparentemente, as arteríolas retinianas não apresentam inervação autônoma simpática. Esta fase pode evoluir diferentemente, caso a PA seja controlada ou não. Se a PA for controlada, a vasoconstrição pode desaparecer. Se a PA não for controlada, poderá ocorrer quebra da barreira hematoretiniana externa, representada pelo epitélio pigmentar da retina e quebra da barreira hemato-retiniana interna, representada pela vasculatura retiniana. Em resposta à $\mathrm{PA}$ elevada e sustentada, ocorre uma aumento do tônus vascular com redução do lúmen vascular. À fase exsudativa segue a fase constritiva. Nesta fase, ocorre o vazamento de plasma e elementos figurados do sangue para dentro da retina. Na região macular poderá formar-se a estrela macular. O extravazamento de sangue, entremeando as fibras nervosas dá o aspecto das hemorragias em chama de vela. As manchas algodonosas surgem em decorrência da não perfusão das fibras nervosas. A fase esclerótica substitui a fase anteriormente mencionada, com os vasos, apresentando alteração da sua coloração, com aspecto de fio de cobre e fio de prata. Isto ocorre devido à esclerose hiperplásica, ao espessamento da túnica média e hiperplasia da túnica muscular. Com a progressão deste processo degenerativo, a parede dos vasos sofre hialinização com perda das células musculares. A fase de complicação da esclerose pode se representar através de microaneurismas, macroaneurismas, oclusão da veia ou artéria central da retina, oclusão de ramo venular e formação de membrana epirretiniana. A remodelagem vascular é comumente encontrada nesta fase, especialmente se a PA estiver controlada.

Os vasos da retina, na HAS, responde com vasoconstrição arteriolar através de mecanismo autorregulatóiro miogênicos e metabólicos. Há fortes indícios de que a vasculatura retiniana não apresenta suprimento inervacional adrenérgico ${ }^{(24-25)}$. As manchas algodonosas são decorrentes da oclusão de arteríolas retinianas terminais, resultando em áreas de isquemia focal de fibras retinianas ${ }^{(26-27)}$.

As alterações vasculares retinianas podem ser sumarizadas em quatro fases em resposta à PA elevada: vasoconstrição intensa, degeneração da musculatura lisa com perda do suporte do endotélio, rotura da barreira endotelial, com passagem do plasma para dentro da parede do vaso, e necrose da mesma, com obliteração da luz do vaso (necrose fibrinóide). Decorrente destas alterações vasculares, são observados os outros achados da RH: manchas algodonosas, exsudato duro, hemorragia retiniana e o papiledema ${ }^{(27-29)}$.

O padrão vascular da coróide em hipertensos foi estuda$\mathrm{do}^{(30)} \mathrm{em}$ material obtido pós-mortem de olho humano, o autor observou: 1) espessamento da adventícia da parede arterial e diminuição da celularidade da camada média da parede do vaso. As veias encontravam-se normais. Registrou ainda que na HAS benigna ocorriam alterações no calibre dos vasos e da celularidade da parede das artérias e arteríolas. Os capilares, as veias e o epitélio pigmentar da retina estavam relativamente inalterados. Na HAS maligna, além da alteração do calibre vascular observado na HAS benigna, as arteríolas e capilares encontravam-se com degeneração hialina e os vasos encontravam-se obliterados e necróticos. A resposta vascular coroidiana ao aumento da PA é controlada, basicamente, pelo sistema nervoso autônomo simpático, com menor participação dos mecanismos autorreguladores locais. Desta forma, um aumento da PA, quando os níveis pressóricos já se encontram elevados, provoca um aumento compensatório do tônus simpático o qual pode provocar lesão na camada muscular e no endotélio vascular. Os achados histológicos vasculares coroidianos, em resposta ao aumento da PA, são a necrose fibrinóide das artérias e arteríolas, além da oclusão da coriocapilar. O epitélio pigmentar da retina sobrejacente torna-se degenerativo, formando as manchas de Elschnig ${ }^{(23)}$. As manchas de Elschnig foram estudadas ${ }^{(31)}$ em macacos Rhesus e registraram as seguintes alterações histopatológicas: obstrução das arteríolas terminais da coróide e da coriocapilar por trombos de fibrina, necrose do epitélio pigmentar da retina e exsudação de fibrina para dentro da membrana de Bruch e sub-retiniano. A coroidopatia hipertensiva foi estudada em modelos animais de hipertensão maligna ${ }^{(32)}$. Sendo os achados, categorizados em três fases principais: a fase isquêmica aguda, a fase oclusiva crônica e a fase crônica reparativa. Estas fases, segundo o pesquisador, apresentaram características histopatológicas distintas. Há um possível envolvimento do sistema renina-angiotensinaaldosterona na fisiopatologia da $\mathrm{CH}^{(3)}$, sendo a angitensina II um poderoso vasoconstritor sobre a vasculatura da coróide. 
Seus achados oftalmoscópicos foram definidos como lesão aguda e crônica do epitélio pigmentar da retina e descolamento seroso da retina.

A fisiopatogenia do envolvimento da cabeça do nervo óptico na HAS continua controversa. Foi observado redução do fluxo axoplasmático na cabeça do nervo óptico em modelos animais, as quais apresentaram papiledema secundário à hipertensão arterial. Os autores ${ }^{(23)}$ sugeriram que tais alterações ocorreram, possivelmente, decorrentes da isquemia do disco óptico. Outros autores ${ }^{(17-18)}$ foram mais enfáticos em seus comentários, e concluíram que o papiledema é decorrente da isquemia do nervo óptico e não da hipertensão intracraniana decorrente da HAS.

\section{MÉTODOS DE INVESTIGAÇÃO DA RETINOPATIA HIPERTENSIVA}

As alterações do fundo de olho que traduzem maior severidade da RH, como as hemorragias, exsudatos e papiledema são raramente vistos ${ }^{(33-34)}$. Talvez este fato se deva ao tratamento precoce e à eficácia das drogas anti-hipertensivas ${ }^{(33)}$. Nestas circunstâncias, a avaliação qualitativa mostra-se imprecisa, e deve-se, portanto, dar maior ênfase às alterações vasculares mais precoces do que ao envolvimento retiniano ${ }^{(34)}$. Os achados do fundo de olho estudados nos pacientes com HAS podem ser acessados por diversas maneiras. A forma mais simples de acessar o fundo de olho é através da oftalmoscopia direta ${ }^{(35)}$. A oftalmoscopia indireta também é um método de investigação das alterações do fundo de olho(4). $\mathrm{O}$ filtro verde, quando utilizado para realizar a oftalmoscopia, permite melhor definição da borda dos vasos ${ }^{(1)}$. Acrescenta-se ainda que o oftalmoscópio direto perde em qualidade por não oferecer ao observador uma visão estereoscópica ${ }^{(1)}$. A visualização do fundo de olho pode ser realizada através de biomicroscopia com lente de três espelhos acoplado a um filtro verde. Tendo a mesma dificuldade em definir o diâmetro do vaso $^{(36)}$, a medida do diâmetro vascular obtido, através de um observador, foi comparado com a de um método computadorizado. Observaram que as medidas da coluna de sangue eram maiores na retinografia observacional quando, comparadas ao método computadorizado, caracterizando um erro metodológico do observador. Alguns autores ${ }^{(22,37-39)}$ avaliaram o fundo de olho por meio da retinografia orientada para determinados segmentos da retina. Uma outra maneira de analisar a imagem do fundo de olho é projetando-se o diapositivo obtido por retinografia sob dois círculos concêntricos, de forma que o disco óptico preencha o círculo menor. Esta condição permite a análise dos vasos na borda do círculo maior, sendo que o círculo menor e o maior apresentam respectivamente raios de 5 e $20 \mathrm{~cm}$. Com esta metodologia, esses autores ${ }^{(40)}$ minimizaram a possibilidade de erro na interpretação da variação do tamanho do disco óptico e estudaram vasos de diâmetro entre 20 a $100 \mu \mathrm{m}$. A angiofluoresceinografia também é uma forma de investigar as alterações do fundo de olho associadas a $\operatorname{HAS}^{(3,18-19,26,31,41-42)}$.
Um aspecto importante são as diferenças nas dimensões dos vasos, quando comparamos a oftalmoscopia ou a retinografia à angiofluoresceinografia. $\mathrm{Na}$ angiofluoresceinografia, os vasos apresentam maior diâmetro. Isto ocorre porque, a fluoresceína mistura-se à coluna líquida do sangue. Na retinografia ou na oftalmoscopia vemos, principalmente, a porção central do vaso onde encontramos, predominantemente, células vermelhas do sangue. Isto torna a borda do vaso imprecisa e o vaso aparentemente mais estreito ${ }^{(20,24)}$. Mais recentemente, métodos computadorizados vêm sendo utilizados para o estudo da microvascularidade retiniana ${ }^{(43-44)}$. Alguns pesquisadores têm investigado as alterações vasculares relacionadas à HAS no leito vascular da conjuntiva bulbar do olho ${ }^{(45-47)}$.

\section{INTERPRETAÇÃO DOS SINAIS DE RETINOPATIA HIPERTENSIVA}

Os vários sinais utilizados como critérios para o diagnóstico e classificação da RH são motivos de controvérsias. Sobre as alterações vasculares, o estreitamento e a retificação arteriolar aparecem, também, com o avançar da idade ${ }^{(1,33)}$. O estreitamento é raro em normotensos e em pessoas com menos de 70 $\operatorname{anos}^{(9)}$. Quando analisado o estreitamento vascular, a primeira dificuldade é descobrir quais são os vasos (arteríolas e vênulas) a serem comparados. Devido a este fato, o calibre vascular não seria um parâmetro adequado, diante das variações anatômicas existentes nos vasos da retina ${ }^{(33)}$. Outro fato relevante na análise da RH é que os sinais são avaliados qualitativamente, permitindo uma ampla interpretação dos achados pelos observadores. Há discordância na interpretação da relação calibre vascular arteríola/vênula pela oftalmoscopia convencional tanto intra-observadores como entre-observadores, tornando-se um critério de difícil interpretação ${ }^{(18,20)}$.

Embora muitos autores aceitem a classificação de KWB bem como a importância desta classificação para prognóstico da $\mathrm{HAS}^{(48)}$, a classificação de KWB nos dá uma falsa impressão de evolução da $\mathrm{RH}^{(24)}$ e apresenta ainda outras limitações ${ }^{(12)}$, como a não distinção adequada entre as alterações hipertensivas e escleróticas vasculares, o grupo I e II da classificação de KWB permite interpretação enganosa, e, às vezes, o paciente não se encaixa em nenhum dos grupos. Há limitação da oftalmoscopia direta e da classificação de KWB na investigação de hipertensos leves e moderados ${ }^{(37)}$. Outras condições que limitam a oftalmoscopia como método para o diagnóstico da RH são o tempo gasto para realizar o exame, a necessidade de um observador com experiência e o fato de ser uma avaliação qualitativa ${ }^{(49)}$. Mais recentemente, como metodologia, o fundo de olho foi avaliado através da oftalmoscopia direta e para classificar as alterações, foi utilizada uma classificação de KWB modificada. Deste estudo foi concluído que o exame do fundo de olho, pela oftalmoscopia, não é um método, na maioria das vezes, preciso para avaliar a severidade da $\mathrm{HAS}^{(50)}$.

O grupo IV da classificação de KWB caracterizado pelos 
sinais dos grupos I, II e III mais papiledema, está relacionado a um prognóstico sombrio da HAS, onde a sobrevida é limita$\mathrm{da}^{(7)}$. Por outro lado, foi sugerido que o papiledema bilateral que acompanha a HAS em pacientes tratados é um sinal limitado para caracterizar um mal prognóstico ${ }^{(51)}$. Há controvérsia quanto aos sinais que revelam o envolvimento do fundo de olho na HAS. Isto é agravado pelo fato de que o médico é induzido a encontrar alterações quando sabe do diagnóstico da $\mathrm{HAS}^{(20)}$. Com o avançar da idade, os vasos sofrem alterações arterioescleróticas (espessamento da parede vascular) que muito lembram as alterações da HAS. Com isto, o diagnóstico da retinopatia hipertensiva fica mais difícil ${ }^{(1,4)}$.

Foi relatado variação entre-observadores de $38 \%$ na interpretação das anormalidades do fundo de olho na HAS através da oftalmoscopia $\operatorname{direta}^{(37)}$. A literatura ${ }^{(20)}$ apresenta dados que reforçam a discordância na interpretação dos achados de fundo de olho intra e entre-observadores. Mais recentemente ${ }^{(52)}$ foi relatado que a RH foi observada em $6 \%$ dos normotensos e o estreitamento arteriolar em $11 \%$ dos normotensos. Em um estudo, onde foram excluídos os pacientes hipertensos, observou-se a presença dos sinais de $\mathrm{RH}$ em pacientes portadores de doença da artéria coronária, e tal correlação foi positi$\mathrm{va}^{(53)}$. Estes dados reforçam a idéia de que os sinais que traduzem o comprometimento do olho, como órgão-alvo da HAS, apresentam baixa precisão.

Os sinais utilizados para o diagnóstico da RH também podem ser observados em outras enfermidades, o que nos obriga a fazer um diagnóstico diferencial. Para exemplificar esta situação, hemorragia e lesões exsudativas podem aparecer em pacientes com Diabetes Melitus e na Síndrome da Imunodeficiência adquirida (AIDS) ${ }^{(1)}$. Os sinais de RH foram observados em pacientes com coartação aórtica ${ }^{(10)}$. Uma série de enfermidades devem ser lembradas diante de um edema bilateral de papila, como: hipertensão intracraniana de causa inflamatória ou tumoral, a infecção do sistema nervoso central, a hemorragia subaracnóide e outras enfermidades ${ }^{(54)}$. No diagnóstico diferencial com a HAS, devemos incluir condições que aumentem a PA, secundariamente, à lesão parenquimatosa renal: pielonefrite crônica, glomerulonefrite crônica, hipoplasia renal, patologias císticas renais e neoplasia ${ }^{(54)}$. Há ainda referências na literatura, caracterizando a regressão dos sinais de $\mathrm{RH}^{(55-56)}$. Por outro lado, já foi observado correlação entre os parâmetros do fundo de olho e os níveis pressóricos, mesmo na utilização de tratamento anti-hipertensivo ${ }^{(57)}$.

\section{PREVALÊNCIA DA RETINOPATIA HIPERTENSIVA}

Os critérios utilizados para a caracterização da amostra influenciam na prevalência da RH. Variando o tamanho da amostra, a raça, a presença e a inclusão de fatores de exclusão como, doenças sistêmicas (doença renal, diabetes mellitus) e/ ou oculares (glaucoma, cicatrizes coriorretinianas) alteram a prevalência da retinopatia hipertensiva. Alguns trabalhos analisam seus dados, considerando ou não o tratamento da
HAS. Outros fatores importantes devem ser considerados: o método de investigação da retinopatia hipertensiva (oftalmoscopia direta, oftalmoscopia indireta, retinografia e angiofluoresceinografia) e o conceito de RH do observador. Os observadores, freqüentemente, desconhecem os dados da história clínica dos pacientes. Utilizando-se da retinografia para avaliar o fundo de olho, e, baseado na classificação de KWB, a prevalência da RH foi de $75 \%$ em seu estudo desenvolvido no Quênia, África ${ }^{(37)}$. Em 350 nigerianos hipertensos, onde os achados do fundo de olho foram abordados pela oftalmoscopia direta e classificados de acordo com a classificação de KWB, a prevalência foi maior de $70 \%{ }^{(58)}$. A RH apresentou maior prevalência em pacientes hipertensos submetidos a tratamento medicamentoso com microalbuminúria persistente ${ }^{(59)}$. A RH esteve presente em $11 \%$ da amostra de um estudo onde o autor analisou seus dados, considerando o tratamento medicamentoso e o controle dos níveis pressóricos ${ }^{(52)}$. Em um estudo, utilizando-se da retinografia e os dados agrupados, segundo a classificação de KWB para o diagnóstico da RH, a prevalência da RH foi de $11 \%$ e $21 \%$ em Europeus e Afrocaribenhos, respectivamente ${ }^{(38)}$. Utilizando-se da angiofluoresceinografia, estudos realizados no Brasil, mostraram a freqüência do diagnóstico da RH: em um estudo com 6.300 exames angiofluoresceinográficos, foram encontrados $2,03 \%$ dos pacientes com $\mathrm{RH}^{(60)}$. Em outro estudo ${ }^{(61)}$, com 1.006 angiofluoresceinografias, a RH apresentou freqüência de $39,5 \%$.

\section{LESÃO DE ÓRG ̃̃OS-ALVO NA HIPERTENSÃO ARTERIAL SISTÊMICA}

Qual dos parâmetros traduziria com maior precisão e mais precocemente a lesão de órgão-alvo na HAS? Foi sugerido que a RH é mais sensível do que a hipertrofia ventricular esquerda (HVE) no reconhecimento da lesão de órgãos-alvo da $\mathrm{HAS}^{(62)}$. A prevalência de doença da artéria coronária foi maior em pacientes com microalbuminúria, persistente ao tratamento da HAS, e, neste mesmo grupo de pacientes, a RH teve maior prevalência ${ }^{(59)}$. Os pacientes com HVE apresentaram anormalidades retinianas mais severas e maior envolvimento renal, e, aqueles hipertensos sem HVE tinham menor dano de outros órgãos-alvo ${ }^{(63)}$. Não foi relatado associação entre os níveis plasmático de sódio, potássio, cloreto e creatinina com as lesões do fundo de olho em modelos animais de HAS aguda ${ }^{(6)}$.

Passados mais de cento e cinqüenta anos da descoberta do oftalmoscópio por Helmholtz, e mesmo com técnicas mais apuradas para a investigação do leito vascular retiniano, persistem dúvidas quanto aos sinais de RH. Estudos recentes ${ }^{(15,36,43)}$ procuram analisar a imagem do fundo de olho por meio de metodologia digitalizada com a utilização de software, que tornam a análise mais precisa e que seja reproduzível. Com isto será possível quantificar melhor a microvascularidade retiniana imprescindível para o estudo da fisiopatologia da HAS, bem como acompanhar o tratamento medicamentoso, e 
para a associação destes dados com os fatores de risco às doenças cardiovasculares. Entre os fatores de risco cardiovasculares temos a obesidade ${ }^{(64)}$ e a $\mathrm{HAS}^{(65)}$. Estudos recentes apontam para uma associação entre leptina e os fatores de risco cardiovasculares anteriormente $\operatorname{citados}^{(66)}$. A leptina está associada também à severidade da $\mathrm{RH}^{(67)}$. Neste contexto de fatores de risco cardiovasculares, Revean descreveu, em 1988, a síndrome X (ou síndrome metabólica) onde um conjunto de enfermidades metabólicas (dislipidemia, obesidade e diabetes mellitus) associado ou não à HAS, poderá ser investigado por métodos quantitativos para a análise do fundo de olho ${ }^{(68)}$. Cabe ainda salientar a importância do estudo do leito vascular no glaucoma. Pesquisas recentes mostram que, a elevação da pressão intra-ocular não é o único mecanismo gerador da perda do campo visual; a isquemia crônica do nervo óptico de primatas induzida por endotelina-1 resultou na perda de axônios sem alterar a pressão intra-ocular ${ }^{(69)}$. Pesquisas que analisam o fluxo vascular retiniano apontam para um fluxo vascular reduzido no glaucoma; assim, drogas vasodilatadoras teriam um papel estratégico no tratamento do mesmo $^{(70)}$.

\section{ABSTRACT}

The present study carries out a review of the theme hypertensive retinopathy. Thus it presents a brief review of the historical data on hypertensive retinopathy. The study reports the classical alterations of hypertensive retinopathy and its classifications, as well as the most recent findings associated with systemic arterial hypertension, the likely pathophysiological mechanisms, and the several methods used for investigation, their advantages and disadvantages; a critical view of the interpretation of signs of the ocular bulb involvement by systemic arterial hypertension; furthermore, based on the diversity of methodologies used in the investigation of retinopathy, comments are made on its reverberation, in the prevalence of hypertensive retinopathy and its implications, as a target organ of systemic arterial hypertension, in an updated context of the metabolic syndrome and of other elements associated with systemic arterial hypertension, such as leptin and endothelin.

Keywords: Hypertension/complications; Retinal diseases/ etiology; Blood pressure; Ophthalmoscopy/methods

\section{REFERÊNCIAS}

1. Coles WH. Hypertension and retinal vessels. Heart Dis Stroke 1994;3:304-8

2. Ellenberger CJ. Ischemic optic neuropathy as a possible early complication of vascular hypertension. Am J Ophthalmol 1979;88:1045-51.

3. Hayreh SS, Servais GE, Virdi PS. Fundus lesions in malignant hypertension: VI. Hypertensive choroidopathy. Ophthalmology 1986;93:1383-400.

4. Matas BR. The Optic fundus and hypertension. Med Clin North Am 1997; 61:547-64

5. Walsh JB. Hypertensive retinopathy: description, classification, and prognosis. Ophthalmology 1982;89:1127-31.
6. Hayreh SS, Servais GE, Virdi PS, Marcus ML, Rojas P, Woolson RF. Fundus lesions in malignant hypertension: III. Arterial blood pressure, biochemical, and fundus changes. Ophthalmology 1985;92:45-59.

7. Keith NM, Wagener HP, Barker NW. Some different types of essential hypertension: their course and prognosis. Am J Med Sci 1939;197:332-43.

8. Gans LJA. Classification of the arteriosclerotic-hypertensive fundus oculi in patients treated with sympathectomy. Arch Ophthalmol 1944;32:267-75.

9. Gelfand M. Hypertensive retinopathy: a suggested modified classification. Cent Afr J Med 1979;25:110-1.

10. Vera TV, Juliá JMG, Romo MCC, Romero MAPJ, Salazar E. Retinopatia hipertensiva en pacientes con coartacion aortica. Arch Inst Cardiol Mex 1988; 58:563-8

11. Heidbreder E, Hüller U, Schäfer B, Heidland A. Severe hypertensive retinopathy. Am J Nephrol 1987; 7:394-400.

12. Hayreh SS. Classification of hypertensive fundus changes and their order of appearance. Ophthalmologica 1989;198:247-60.

13. Mcmahon TT, Maino JH. Hypertensive choroidopathy. J Am Optom Assoc 1986;53:713-7.

14. Kishi S, Tso MO, Hayreh SS. Fundus lesions in malignant hypertension: I. A pathologic study of experimental hypertensive choroidopathy. Arch Ophthalmol 1985; 103:1189-97.

15. Brinchmann-Hansen O, Christensen CC, Myhre K. The response of the light reflex of retinal vessels to reduced blood pressure in hypertensive patients. Acta Ophthalmol 1990;68:155-61.

16. Parr JC. Hypertensive generalized narrowing of retinal arteries. Trans Ophthalmol Soc N Z 1974;26:55-60.

17. Kishi S, Tso MO, Hayreh SS. Fundus lesions in malignant hypertension: II. A pathologic study of experimental hypertensive optic neuropathy. Arch Ophthalmol (Copenh)1985;103:1198-206.

18. Hayreh SS, Servais GE, Virdi PS. Fundus lesion in malignant hypertension: V. Hypertensive optic neuropathy. Ophthalmology 1986;93:74-87.

19. Hayreh SS, Servais GE, Virdi PS. Fundus lesions in malignant hypertension: IV. Focal intraretinal periarteriolar transudates. Ophthalmology 1986;92:60-73.

20. Hayreh SS, Servais GE, Virdi PS. Retinal arteriolar changes in malignant arterial hypertension. Ophthalmologica 1989;198:178-96.

21. Stanton AV, Wasan B, Cerutti A, Ford S, Marsh R, Sever PP, Thom AS, Hughes AD. Vascular network changes in the retina with age and hypertension. J Hypertens 1995;13:1724-8.

22. Yu T, Mitchell P, Berry G, Li W, Wang JJ. Retinopathy in older persons without diabetes and its relationship to hypertension. Arch Ophthalmol 1998; 116:83-9.

23. Tso MOM, Jampol LM. Pathophysiology of hypertensive retinopathy. Ophthalmology 1982;89:1132-45.

24. Hayreh SS. Hypertensive retinopathy: introduction. Ophthalmologica 1989; 198:173-7.

25. Ramalho PS, Dollery CT. Hypertensive retinopathy: caliber changes in retinal blood vessels following blood-pressure reduction and inhalation of oxygen. Circulation 1968;37:580-8.

26. Hayreh SS, Servais GE, Virdi PS. Cotton-wool spots (inner retinal ischemic spots) in malignant arterial hypertension. Ophthalmologica 1989;198:197-215.

27. Ashton N. The eye in malignant hypertension. Trans Am Acad Ophthalmol Otolaryngol 1972;76:17-40.

28. Garner A, Ashton N. Pathogenesis of hypertensive retinopathy: a review. J R Soc Med 1979;72:362-5.

29. Garner A, Ashton N, Tripathi R, Kohner EM, Bulpitt CJ, Dollery CT. Pathogenesis of hypertensive retinopathy: An experimental study in the monkey. Br J Ophthalmol 1975;59:3-44

30. Friedman E, Smith TR, Kuwabara T, Beyer CCK. Choroidal vascular patterns in hypertension. Arch Ophthalmol 1964;71:842-50.

31. De Venicia G, Wallow I, Houser D, Wahlstrom M. The eye in accelerated hypertension: I. Elschnig's spots in nonhuman primates. Arch Ophthalmol 1980;98:913-8.

32. Kishi S, Tso MOM, Hayreh SS. Fundus lesions in malignant hypertension: I. A pathologic study of experimental hypertensive choroidopathy. Arch Ophthalmol 1985;103:1189-97.

33. Stokoe NL, Turner RW. Normal retinal vascular pattern: arteriovenous ratio as a measure of arterial calibre. Br J Ophthalmol 1966;50:21-40.

34. King LA, Stanton AV, Sever PS, Thom SA, Hughes AD. Arteriolar lengthdiameter (L:D) ratio: a geometric parameter of the retinal vasculature diagnostic of hypertension. J Hum Hypertens 1996;10:417-8.

35. Pontremoli R, Viazzi F, Sofia A, Tomolillo C, Ruello N, Bezante GP et al. Microalbuminuria: a marker of cardiovascular risk and organ damage in essential hypertension. Kidney Int 1997; Suppl 63:S163-5. 
36. Newsom RS, Sullivan PM, Rassam SM, Jagoe R, Kohner EM. Retinal vessel measurement: comparison between observer and computer driven methods. Graefes Arch Clin Exp Ophthalmol 1992;230:221-5.

37. Dimmitt SB, Eames SM, Gosling P, West JN, Gibson JM, Littler WA. Usefulness of ophthalmoscopy in mild to moderate hypertension. Lancet 1989;1:1103-6

38. Sharp PS, Chaturvedi N, Wormald R, Mckeigue PM, Marmot MG, Young SM. Hypertensive retinopathy in afro-caribbeans and europeans: prevalence and risk factor relationships. Hypertension 1995;25:1322-5.

39. Awan AM, Ojiambo HP, Ogada T. Hypertensive retinopathy. A prospective hospital study of 100 hypertensive Kenyan Africans. East Afr Med J 1974;51:304-20.

40. Stanton AV, Mullaney P, Mee F, O'Brien ET, O'Malley K. A method of quantifying retinal microvascular alterations associated with blood pressure and age. J Hypertens 1995; 13:41-8.

41. Lafaut BA, De Vriese AS, Stulting AA. Fundus fluorescein angiography of patients with severe hypertensive nephropathy. Graefes Arch Clin Exp Ophthalmol 1997;235:749-54

42. Wolf S, Arend O, Schulte K, Ittel TH, Reim M. Quatification of retinal capillary density and flow velocity in patients with essential hypertension. Hypertension 1994;23:464-7.

43. Blum M, Bachmann K, Wintzer D, Riemer T, Vilser W, Strobel J. Noninvasive measurement of the bayliss effect in retinal autoregulation. Graefes Arch Clin Exp Ophthalmol 1999;237:296-300.

44. Kutschbach P, Wolf S, Sieveking M, Ittel TH, Schulte K, Reim M. Retinal capillary density in patients with arterial hypertension: 2-year follow-up. Graefes Arch Clin Exp Ophthalmol 1996;236:410-4.

45. Lack A, Adolph W, Ralston W, Leiby G, Winsor T, Griffith G. Biomicroscopy of conjunctival vessels in hypertension. Am Heart J 1949;38:654-64.

46. Landau J, Davis E. Capillary thinning and high capillary blood-pressure in hypertension. Lancet 1957;1327-30.

47. Körber N, Jung F, Kiesewetter H, Wolf S, Prünte C, Reim M. Microcirculation in the conjunctival capillaries of healty and hypertensive patients. Klin Wochenschr 1986;64:943-5.

48. Breslin DJ, Gifford RWJ, Fairbairn JF, Kearns TP. Prognostic importance of ophthalmoscope findings in essential hypertension. JAMA 1966;195:335-8.

49. Thien T Lenders JW. Hypertension and target organ damage. Neth J Med 1995;47:141-4.

50. Fuchs FD, Maestri MK, Bredemeier M, Cardozo SE, Moreira FC, Wainstein MV et al. Study of the usefulness of optic fundi examination of patients with hypertension in a clinical setting. J Hum Hypertens 1995;9:547-51.

51. McGregor E, Isles CG, Jay JL, Lever AF, Murray GD. Retinal changes in malignant hypertension. Br Med J 1986;292:233-4

52. Klein R, Klein BE, Moss SE, Wang Q. Hypertension and retinopathy, arteriolar narrowing, and arteriovenous nicking in a population. Arch Ophthalmol $1994 ; 112: 92-8$
53. Michelson EL, Morganroth J, Nichols CW, MacVaugh H. Retinal arteriolar changes as an indicator of coronary artery disease. Arch intern Med 1979; 139:1139-41

54. Leavitt JA, Pruthi S, Morgenstern BZ. Hypertensive retinopathy mimicking neuroretinitis in a twelve-year-old girl. Surv Ophthalmol 1997;41:477-80.

55. Lowenthal MN, Zimlichman R. Resolution of hypertensive retinopathy despite persistent high diastolic pressure. South Med J 1993;86:190-3.

56. Çakmakçi S, Kadayifçilar S, Aydin P, Bilgin N. Dramatic regression of hypertensive retinopathy following renal transplantation: A Case report. Transplant Proc 1998;30:788-9.

57. Sharrett AR, Hubbard LD, Cooper LS, Sorlie PD, Brothers RJ, Nieto FJ, Pinsky JL, Klein R. Retinal arteriolar diameters and elevated blood pressure: the atherosclerosis risk in communities study. Am J Epidemiol 1999;150:263-70.

58. Ladipo GO. Hypertensive retinopathy in Nigerians: a prospective clinical study of 350 cases. Trop Geogr Med 1981;33:311-6.

59. Biesenbach G, Zazgornik J. High prevalence of hypertensive retinopathy and coronary heart disease in hypertensive patients with persistent microalbuminuria under short intensive antihypertensive therapy. Clin Nephrol 1994;41:211-8.

60. Lucena DR, Siqueira RC. Os dez mais freqüentes diagnósticos angiofluoresceinográficos de um serviço de referência em retina e vítreo. Rev Bras Oftalmol 2000;59:594-6.

61. Veloso JCB, Ventura AG, Escarião PHG, Neto AQL, Dias S. Angiofluoresceinografia em serviço de referência: freqüência diagnóstica. An Fac Med Univ Fed Pernambuco 2001;46:45-7.

62. De Leonardis V, Becucci A, De Scalzi M, Cinelli P. Low incidence of cardiac hypertrophy in essential hypertensives with no retinal changes. Int $\mathrm{J}$ Cardiol 1992;35:95-9.

63. Shigematsu Y, Hamada M, Mukai M, Matsuoka H, Sumimoto T, Hiwada K. Clinical evidence for an association between left ventricular geometric adaptation and extracardiac target organ damage in essential hypertension. J Hypertens 1995; 13:155-60.

64. Manson JE, Willet WC, Stamper MJ, Colditz GA, Hunter DJ, Hankinson SE et al. Body weight and mortality among women. N Engl J Med 1995;333:677-85.

65. Kannel WB, Gordon T, Schwartz MJ. Systolic versus diastolic blood pressure and risk of coronary heart disease. Am J Cardiol 1971;27:335-46.

66. Müller-Wieland D, Kotzka J, Knebel B, Krone W. Metabolic syndrome and hypertension: pathophysiology and molecular basis of insulin resistance. Basic Res Cardiol 1998; 93 Suppl 2:131-4.

67. Uckaya G, Ozata M, Sonmez A, Kinalp C, Eyileten T, Bingol N et al. Is leptin associated with hypertensive retinopathy? J Clin Endocrinol Metab 2000;85:683-7.

68. Reaven GM. Role of insulin resistence in human disease. Diabetes 1988; 37:1595-607.

69. Cioffi GA, Sullivan P. The effect of chronic ischemia on the primate optic nerve. Eur J Ophthalmol 1999;9 Suppl 1:S34-6.

70. Martinez A, Gonzalez F, Capeans C, Perez R, Sanchez-Salorio M. Dorzolamide effect on ocular blood flow. Invest Ophthalmol Vis Sci 1999;40:1270-5.

\section{$6^{\circ}$ CURSO CLEBER GODINHO DE LENTES DE CONTATO}

\section{8 a 23 de Outubro de 2002 \\ BELO HORIZONTE - MG}

INFORMAÇÕES: Consult Comunicações e Eventos

Fone/Fax: (31) 3274-1550

E-mail: comunica@consultcom.com.br

Home-page: www.clebergodinho.med.br 